\title{
Telemetry Discrepancy
}

National Cancer Institute

\section{Source}

National Cancer Institute. Telemetry Discrepancy. NCI Thesaurus. Code C62830.

Problem associated with variability of the transmission of telemetry signals. 